\title{
The initial helium abundance of the Galactic globular cluster system ${ }^{\star}$
}

\author{
M. Salaris ${ }^{1,2}$, M. Riello ${ }^{3,4}$, S. Cassisi ${ }^{5}$, and G. Piotto ${ }^{3}$ \\ 1 Astrophysics Research Institute, Liverpool John Moores University, Twelve Quays House, Egerton Wharf, Birkenhead, \\ CH41 1LD, UK \\ 2 Max-Planck-Institut für Astrophysik, Karl-Schwarzschild-Strasse 1, 85758 Garching, Germany \\ 3 Dipartimento di Astronomia, Vicolo dell'Osservatorio 2, 35122 Padova, Italy \\ e-mail: piotto,riello@pd.astro.it \\ ${ }^{4}$ INAF - Osservatorio Astronomico di Padova, vicolo dell’Osservatorio 5, 35122 Padova, Italy \\ 5 INAF - Osservatorio Astronomico di Collurania, via M. Maggini, 64100 Teramo, Italy \\ e-mail: cassisi@te.astro.it
}

Received 31 October 2003 / Accepted 17 March 2004

\begin{abstract}
In this paper we estimate the initial He content in about $30 \%$ of the Galactic globular clusters (GGCs) from new star counts we have performed on the recently published HST snapshot database of Colour Magnitude Diagrams (Piotto et al. 2002). More specifically, we use the so-called $R$-parameter and estimate the He content from a theoretical calibration based on a recently updated set of stellar evolution models. We performed an accurate statistical analysis in order to assess whether GGCs show a statistically significant spread in their initial He abundances, and whether there is a correlation with the cluster metallicity. As in previous works on the subject, we do not find any significant dependence of the He abundance on the cluster metallicity; this provides an important constraint for models of Galaxy formation and evolution. Apart from GGCs with the bluest Horizontal Branch morphology, the observed spread in the individual helium abundances is statistically compatible with the individual errors. This means that either there is no intrinsic abundance spread among the GGCs, or that this is masked by the errors. In the latter case we have estimated a firm $1 \sigma$ upper limit of 0.019 to the possible intrinsic spread. In case of the GGCs with the bluest Horizontal Branch morphology we detect a significant spread towards higher abundances inconsistent with the individual errors; this can be fully explained by additional effects not accounted for in our theoretical calibrations, which do not affect the abundances estimated for the clusters with redder Horizontal Branch morphology. In the hypothesis that the intrinsic dispersion on the individual $\mathrm{He}$ abundances is zero, taking into account the errors on the individual $R$-parameter estimates, as well as the uncertainties on the cluster metallicity scale and theoretical calibration, we have determined an initial He abundance mass fraction $Y_{\mathrm{GGC}}=0.250 \pm 0.006$. This value is in perfect agreement with current estimates based on Cosmic Microwave Background radiation analyses and cosmological nucleosynthesis computations.
\end{abstract}

Key words. Galaxy: globular clusters: general - Galaxy: evolution - stars: evolution - stars: Population II

\section{Introduction}

The determination of the initial $\mathrm{He}$ abundance of Galactic Globular Cluster (GGC) stars plays an important role in astrophysics, because of its wide-ranging implications.

First of all, due to the fact that GGC stars are the oldest objects in the Galaxy, their initial He abundance $\left(Y_{\mathrm{GGC}}\right.$, where $Y$ denotes the mass fraction of $\mathrm{He}$ ) should mirror the primordial

Send offprint requests to: $\mathrm{M}$. Salaris,

e-mail: ms@astro.livjm.ac.uk

* Based on observations with the NASA/ESA Hubble Space Telescope, obtained at the Space Telescope Science Institute, which is operated by AURA, Inc., under NASA contract NAS5-26555, and on observations retrieved with the ESO ST-ECF Archive.
He abundance $\left(Y_{\mathrm{p}}\right)$ produced during the big bang nucleosynthesis. Secondly, the presence (or absence) of gradients in $Y_{\mathrm{GGC}}$ as a function of the cluster metallicity provides information about Galactic nucleosynthesis and evolution. Thirdly, the existence of an abundance spread at constant metallicity would strenghten the case for He being the so-called "second parameter" (beside metallicity) that determines the morphology of the Horizontal Branch (HB) in the GGC Colour-MagnitudeDiagrams (CMDs - Sandage \& Wildey 1967).

Empirical estimates of $Y_{\mathrm{GGC}}$, are necessarily indirect, since He lines are not detectable in GGC star spectra, apart from the case of hot HB objects, whose atmospheres are however affected by gravitational settling and radiative levitation, which strongly alter their initial chemical stratification (see, e.g., 
Michaud et al. 1983). $Y_{\mathrm{GGC}}$ estimates take advantage of results from stellar evolution theory, which show that the evolution of low mass Population II stars is affected by the initial He content. The so-called $R$-parameter (Iben 1968; Buzzoni et al. 1983; Caputo et al. 1987), defined as the number ratio of HB to Red Giant Branch (RGB) stars brighter than the HB level ( $\left.R=N_{\mathrm{HB}} / N_{\mathrm{RGB}}\right)$, is employed to determine $Y_{\mathrm{GGC}}$. At a given metallicity, a higher initial He-content implies a brighter $\mathrm{HB}$ and, in turn, a lower value of $N_{\mathrm{RGB}}\left(N_{\mathrm{HB}}\right.$ is only slightly affected), with the consequent increase of $R$. Other parameters derived from stellar evolution can also be employed (see, e.g., the discussions in Sandquist 2000; Zoccali et al. 2000), but they are better suited to determine relative He abundances than absolute ones.

In Cassisi et al. (2003, hereafter Paper I) we have shown how $R$-parameter determinations in two samples of GGCs (Sandquist 2000, 43 objects; Zoccali et al. 2000, 26 objects), coupled with a theoretical calibration obtained from updated stellar evolution models, provide a constant value of $Y_{\mathrm{GGC}}$, with no trend with respect to $[\mathrm{Fe} / \mathrm{H}]$. The weighted average of the individual cluster abundances provides $Y_{\mathrm{GGC}}=0.244 \pm 0.006$ or $Y_{\mathrm{GGC}}=0.243 \pm 0.006$, the negligible difference being due to the choice of the observational sample. This value of $Y_{\mathrm{GGC}}$ is in agreement - within the errors - with recent determinations of the cosmological baryonic matter density $\left(\Omega_{b}\right)$ from the Cosmic Microwave Background (CMB) power spectrum obtained by the BOOMERANG, DASI, MAXIMA and WMAP experiments (e.g. Pryke et al. 2002; Ödman et al. 2003; Sievers et al. 2003; Spergel et al. 2003) which, coupled to BBN calculations (Burles et al. 2001), do consistently provide $Y_{\mathrm{p}}=$ $0.248 \pm 0.001$. It is important to recall that earlier studies (Sandquist 2000; Zoccali et al. 2000) that employed older generations of stellar models, found $Y_{\mathrm{GGC}} \sim 0.20$, in complete disagreement with the $\mathrm{CMB}$ constraint and spectroscopic determinations from HII regions $\left(Y_{\mathrm{p}}=0.234 \pm 0.002\right.$ according to Olive et al. $1997 ; Y_{\mathrm{p}}=0.244 \pm 0.002$ according to Izotov \& Thuan 1998).

As for the abundance spread at a given $[\mathrm{Fe} / \mathrm{H}]$, the results of Paper I were contradictory, in the sense that $R$-parameter values from the Zoccali et al. (2000) sample show a spread that is completely consistent with the measurement errors, whereas the sample by Sandquist (2000) shows an intrinsic additional $1 \sigma$ spread in the He abundance of $\sim 0.03$.

In this paper we measured new $R$-parameter values for a sample of 57 GGCs from the database of HST GGC observations by Piotto et al. (2002). We use the HST flight photometric system (without reddening corrections) instead of the Johnson photometric bands. This approach avoids potential subtle errors in the estimate of the visual magnitudes of the HB. In fact, the transformation to the Johnson system requires the knowledge of the cluster reddening, therefore the accuracy of the observed Johnson magnitudes depends on the accuracy of the cluster reddening estimate.

Our determinations of $R$-parameter values constitute the largest existing homogeneous database, encompassing about $30 \%$ of the total GGC population. It is therefore extraordinarily well suited to address in more detail the question of the intrinsic spread in the individual GGC He abundances, and the ratio of He enrichment with metallicity enrichment $(\Delta Y / \Delta Z)$. With regard to the latter quantity, its evaluation is extremely important because of the connections with stellar yields and mechanism of formation of the GGCs, and the determination of their absolute ages. In addition, it is relevant to make a comparison with the value $\Delta Y / \Delta Z$ between $\approx 6$ and 12 (depending on the sample adopted, and with the assumption that the Oxygen abundance is a good tracer of total metallicity) estimated by Olive et al. (1997) for extragalactic HII regions, and the value $\sim 3$ estimated by Izotov \& Thuan (2003). We will also address the hypothesis put forward by D'Antona et al. (2002), which suggests the existence of an He enriched stellar component within individual clusters, due to chemical pollution by the ejecta of massive asymptotic giant branch stars; in particular, we will assess if this phenomenon might seriously affect our determination of $Y_{\mathrm{GGC}}$.

In Sect. 2 we briefly describe the cluster sample and the theoretical models employed. Section 3 presents our results for the $R$-parameter measurements and the determination of $Y_{\mathrm{GGC}}$. A summary and conclusions follow in Sect. 4.

\section{Cluster sample and theoretical stellar models}

We have employed our large photometric database of 74 GGCs observed in the HST $B(F 439 W)$ and $V(F 555 W)$ bands with the WFPC2 (Piotto et al. 2002). The observations, preprocessing, photometric reduction and calibration of the instrumental magnitudes to the HST flight system, as well as the artificial star experiments performed to derive the star count completeness are described in Piotto et al. (2002). For each cluster we measured a number of stars that ranges from a few thousand to $\approx 47000$ (in case of NGC 6388). For 57 of our clusters we have been able to measure $R$. The empirical definition of $R$ is the same as in Zoccali et al. (2000), i.e., the number of RGB stars is computed starting from the level of the observed $F 555 \mathrm{~W}$ magnitude of the Zero Age HB (ZAHB; the lower envelope of the observed HB star distribution). The determination of the ZAHB level has been discussed in Riello et al. (2003) and Recio-Blanco et al. (2004, in preparation). The error on the observed $R$ values is computed by combining in quadrature the Poisson error associated with the measurement of $N_{\mathrm{HB}}$ and $N_{\mathrm{RGB}}$, and the contribution due to the uncertainty on the ZAHB level (which translates into an additional uncertainty on $N_{\mathrm{RGB}}$, hence on $R$ ). Usually, this second contribution is much smaller than the Poisson error associated with the number counts $N_{\mathrm{HB}}$ and $N_{\mathrm{RGB}}$. As for the GGC [Fe/H] values, we take into account current uncertainties on this quantity by employing both the Carretta \& Gratton (1997 - CG97) and Zinn \& West (1984 - ZW84) scales (we assumed an error of \pm 0.15 dex on the individual abundances). The observational data relevant to our analysis are summarized in Table 2.

The theoretical models and isochrones needed to calibrate the relationship between $R$ and $Y$ are the same as in Paper I, computed for a range of $[\mathrm{Fe} / \mathrm{H}]$ and $Y$ values. A full description of these models will be presented in a forthcoming paper (Pietrinferni et al. 2004, submitted to ApJ) however, the basic physical ingredients relevant for the computation of the theoretical $R$ values have already been discussed in Paper I. 
Table 1. Comparison of the ZAHB levels, He core masses (in solar mass units), $\mathrm{HB}$ evolutionary times (in Myr) and RGB evolutionary times at brighter magnitudes than the ZAHB (in Myr) between the models used in Zoccali et al. (2000), in Paper I, and the models by Cassisi et al. (1998), for two selected metallicities (see text for details).

\begin{tabular}{lcccc}
\hline \hline Zoccali et al. (2000) & $M_{V}(\mathrm{ZAHB})$ & $M_{\mathrm{c}}(\mathrm{He})$ & $t_{\mathrm{HB}}$ & $t_{\mathrm{RGB}}$ \\
\hline$Z=0.001$ & 0.54 & 0.499 & 102 & 63 \\
$Z=0.006$ & 0.72 & 0.493 & 112 & 55 \\
\hline Cassisi et al. (1998) & & & & \\
\hline$Z=0.001$ & 0.49 & 0.500 & 85 & 58 \\
$Z=0.006$ & 0.68 & 0.493 & 94 & 47 \\
\hline Paper I & & & & \\
\hline$Z=0.001$ & 0.58 & 0.495 & 88 & 65 \\
$Z=0.006$ & 0.74 & 0.489 & 93 & 56 \\
\hline
\end{tabular}

Here, we briefly recall that our models have been computed with the $\alpha$-enhanced metal distribution given in Salaris \& Weiss (1998), with $\langle[\alpha / \mathrm{Fe}]\rangle=0.4$. The radiative opacities (Alexander \& Ferguson 1994; Iglesias \& Rogers 1996) have been computed specifically for our adopted metal mixture, and the electron conduction opacities are from Potekhin (1999). The nuclear reaction rates from the NACRE database (Angulo et al. 1999) have been employed, with the exception of the ${ }^{12} \mathrm{C}(\alpha, \gamma){ }^{16} \mathrm{O}$ reaction. For this reaction we employ the more accurate recent determination by Kunz et al. (2002), based on $\gamma$ angular distribution measurements of ${ }^{12} \mathrm{C}(\alpha, \gamma){ }^{16} \mathrm{O}$ and a consistent R-matrix analysis of the process. The claimed relative uncertainty of this new rate is half of the uncertainty quoted in previous determinations. The relevant energy loss rates from plasma-neutrino processes have been taken from Haft et al. (1994). We have used the equation of state by Irwin et al. (2004, in preparation) $)^{1}$ computed for our actual $\alpha$-enhanced metal mixture.

Table 1 compares, as an example, the adopted ZAHB level and corresponding He core mass, HB and RGB evolutionary times entering our present theoretical calibration of the $R$-parameter, with the calibration by Zoccali et al. (2000), for $Y=0.245$ and two selected metallicities. The data for the Zoccali et al. (2000) model have been obtained by interpolating within their computed grid, since they did not specifically compute models for $Y=0.245$. By simply comparing the evolutionary timescales that enter the $R$ calibration one notices a reduction of $\sim 20 \%$ of the value of $R$ when passing from Zoccali et al. (2000) models to our new ones, as discussed in Paper I. As a further comparison to highlight the change in the theoretical calibration of $R$ due to the improvements in stellar models, we also display in the same table analogous data from Cassisi et al. (1998) computations, for $Y=0.245$. These models were a sort of intermediate step between the models used in Zoccali et al. (2000) and in Paper I, and have never been used to determine $Y_{\mathrm{GGC}}$. A calibration based on Cassisi et al. (1998) models reduces the value of $R$ by $\sim 10 \%$ at $Z=0.001$ with respect to

1 The equation of state code is made publicly available at ftp://astroftp.phys.uvic.ca/pub/irwin/eos/code/ eos_demo_fortran.tar.gz
Zoccali et al. (2000), whereas at $Z=0.006$ the reduction is by only about $2 \%$.

The bolometric luminosities and effective temperatures of the models have been converted to the HST flight system using the transformations by Origlia \& Leitherer (2000), based on the model atmospheres by Bessel et al. (1998). In Paper I we have also shown how our treatment of the semiconvective regions during the He burning phase does agree with independent observational constraints coming from the $R_{2}$ parameter measured in a sample of GGCs by Sandquist (2000 - see Paper I for details).

The theoretical values of $R$ are computed by considering the RGB evolutionary time of the RGB mass populating the relevant isochrone, and the HB lifetime of a star populating the middle of the RR Lyrae instability strip $\left(\log \left(T_{\text {eff }}\right)=3.85\right)$. This is strictly adequate only for those clusters with an HB populated at the RR Lyrae instability strip and redward (increasing total stellar mass), since the HB evolutionary timescales are basically unchanged when moving from the instability strip towards the red (see Paper I, and references therein). However, stars populating the bluer part of the HB do show different evolutionary times, which increase for decreasing total stellar mass (bluer colour). At the bluest end of a typical HB the increase of the HB evolutionary time with respect to the RR Lyrae strip counterpart can amount to about $20 \%$ (see, e.g., Zoccali et al. 2000).

\subsection{The effect of He enriched subpopulations in individual clusters}

Another effect that might bias our determination of $Y_{\mathrm{GGC}}$ is the He enrichment in some GGC proposed by D'Antona et al. (2000). More in detail, to explain the observed CNO abundance anomalies and the extended blue $\mathrm{HB}$ tails in some GGCs, D'Antona et al. (2002) suggested the existence of an He enriched stellar component within the clusters. This enrichment is due to chemical pollution by the ejecta of massive asymptotic giant branch stars belonging to the cluster; this chemical pollution may explain the mentioned $\mathrm{CNO}$ anomalies and HB colours. In Riello et al. (2003) we showed that this supposed enrichment does not alter the brightness of the RGB bump in the luminosity function of GGCs. Here we estimate the effect on the He abundance estimated through the $R$ parameter. As in Riello et al. (2003), and similarly to D'Antona et al. (2002), we considered a cluster stellar population composed of $64 \%$ of stars with a given initial He abundance as adopted in our models - here denoted as "normal" - , and $36 \%$ of stars with an initial $Y$ randomly distributed between the "normal" value and a mass fraction 0.06 larger. In general, for any morphology of the HB, the level of the HB that enters the definition of $R$ is still determined by the $64 \%$ of objects with "normal" abundance; $N_{\mathrm{HB}}$ for the whole population tends to increase, whereas $N_{\mathrm{RGB}}$ decreases, increasing the helium content estimate. When using our present standard calibration of $R$ as a function of $Y$ in He-enhanced clusters, we would overestimate the initial He mass fraction by at most $0.005-0.006$ for clusters where the HB instability strip and the region redwards of this 


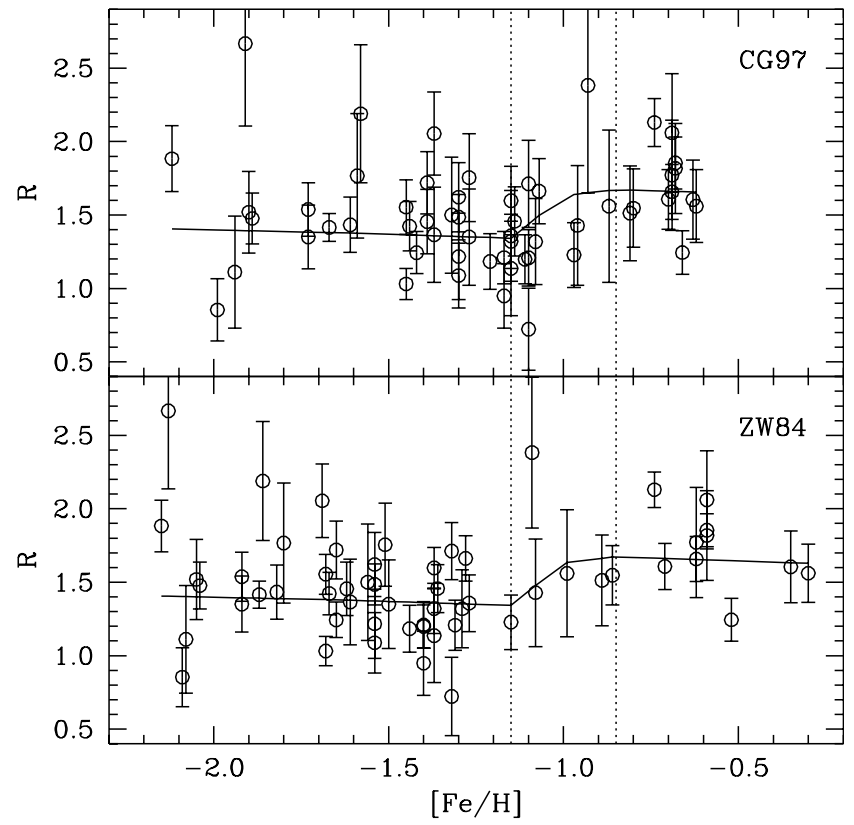

Fig. 1. Observed $R$-parameter values as a function of the cluster $[\mathrm{Fe} / \mathrm{H}]$ for the two adopted metallicity scales. The theoretical calibration for $Y=0.245$ and a cluster age of $13 \mathrm{Gyr}$ is also shown.

strip are populated, whereas for blue clusters the effect is an overestimate of at most $0.01-0.02$. Most probably the degree of He enrichment (if it is real) will vary from cluster to cluster (for the clusters affected by this phenomenon), and the real overestimate will be between zero and the values quoted above.

\section{The value of $Y_{\mathrm{GGC}}$}

Figure 1 displays the observed $R$ values as a function of the cluster metallicities, on both the CG97 and ZW84 metallicity scales. The solid line denotes the theoretical values computed for an age of $13 \mathrm{Gyr}$ and an initial $Y=0.245$. As already discussed in Paper $I$, theoretical models predict an almost constant trend of $R$ with $[\mathrm{Fe} / \mathrm{H}]$, with a discontinuity located between $[\mathrm{Fe} / \mathrm{H}] \sim-1.15$ and $[\mathrm{Fe} / \mathrm{H}] \sim-0.85$. The abrupt increase of $R$ in this metallicity range is due to the fact that the RGB bump, previously located at brightnesses larger than the ZAHB, moves below the ZAHB level with increasing metallicity, thus causing a decrease in the number of RGB stars brighter than the ZAHB (see, e.g., Salaris et al. 2002). As explained in Paper I, it is only in this metallicity range that the theoretical calibration of $R$ as a function of $Y$ does depend on the assumed age, due to the fact that the RGB bump brightness is also a function of age. The net effect is to decrease the theoretical value of $R$ at a given $Y$ when the age decreases.

Outside this narrow metallicity range a variation of the age between, i.e. $14 \mathrm{Gyr}$ and $8 \mathrm{Gyr}$, produces a change in the inferred value of $Y$ by less than 0.001 . This is why in this case we can safely neglect the precise individual values of the cluster ages in this metallicity range, and just assume a common reasonable age. Just to show the consistency of our GGC isochrone ages with independent estimates of the age of the
Table 2. Cluster data. The columns display, respectively, cluster name, measured value of $R$ with associated error, $[\mathrm{Fe} / \mathrm{H}]$ on the $\mathrm{ZW} 84$ and CG97 scale, and value of $H B_{\text {type }}$ (see text for details).

\begin{tabular}{|c|c|c|c|c|c|}
\hline Cluster & $R$ & $\sigma(R)$ & {$[\mathrm{Fe} / \mathrm{H}]_{\mathrm{ZW} 84}$} & {$[\mathrm{Fe} / \mathrm{H}]_{\mathrm{CG} 97}$} & $H B_{\text {type }}$ \\
\hline IC 4499 & 1.351 & 0.302 & -1.50 & -1.27 & 0.11 \\
\hline NGC 104 & 1.607 & 0.157 & -0.71 & -0.70 & -0.99 \\
\hline NGC 362 & 1.358 & 0.193 & -1.27 & -1.15 & -0.87 \\
\hline NGC 1261 & 1.208 & 0.170 & -1.31 & -1.10 & -0.71 \\
\hline NGC 1851 & 1.457 & 0.163 & -1.36 & -1.14 & -0.36 \\
\hline NGC 1904 & 2.055 & 0.251 & -1.69 & -1.37 & 0.89 \\
\hline NGC 2808 & 1.598 & 0.139 & -1.37 & -1.15 & -0.49 \\
\hline NGC 3201 & 1.136 & 0.319 & -1.37 & -1.15 & 0.08 \\
\hline NGC 4147 & 1.767 & 0.408 & -1.80 & -1.59 & 0.55 \\
\hline NGC 4372 & 1.111 & 0.366 & -2.08 & -1.94 & 1.00 \\
\hline NGC 4590 & 0.854 & 0.200 & -2.09 & -1.99 & 0.17 \\
\hline NGC 4833 & 2.189 & 0.405 & -1.86 & -1.58 & 0.93 \\
\hline NGC 5024 & 1.477 & 0.159 & -2.04 & -1.89 & 0.81 \\
\hline NGC 5634 & 1.433 & 0.184 & -1.82 & -1.61 & 0.91 \\
\hline NGC 5694 & 1.537 & 0.167 & -1.92 & -1.73 & 1.00 \\
\hline NGC 5824 & 1.415 & 0.092 & -1.87 & -1.67 & 0.79 \\
\hline NGC 5904 & 1.199 & 0.146 & -1.40 & -1.11 & 0.31 \\
\hline NGC 5927 & 1.561 & 0.199 & -0.30 & -0.62 & -1.00 \\
\hline NGC 5946 & 1.321 & 0.171 & -1.37 & -1.15 & 0.71 \\
\hline NGC 5986 & 1.423 & 0.144 & -1.67 & -1.44 & 0.97 \\
\hline NGC 6093 & 1.031 & 0.144 & -1.67 & -1.44 & 0.97 \\
\hline NGC 6139 & 1.244 & 0.121 & -1.65 & -1.42 & 0.91 \\
\hline NGC 6171 & 1.560 & 0.432 & -0.99 & -0.87 & -0.73 \\
\hline NGC 6205 & 1.719 & 0.197 & -1.65 & -1.39 & 0.97 \\
\hline NGC 6218 & 1.366 & 0.292 & -1.61 & -1.37 & 0.97 \\
\hline NGC 6229 & 1.485 & 0.140 & -1.54 & -1.30 & 0.24 \\
\hline NGC 6235 & 0.949 & 0.218 & -1.40 & -1.17 & 0.89 \\
\hline NGC 6266 & 1.662 & 0.153 & -1.28 & -1.07 & 0.32 \\
\hline NGC 6273 & 1.554 & 0.136 & -1.68 & -1.45 & 0.97 \\
\hline NGC 6284 & 1.210 & 0.157 & -1.40 & -1.17 & 0.83 \\
\hline NGC 6287 & 1.519 & 0.273 & -2.05 & -1.90 & 0.98 \\
\hline NGC 6293 & 1.351 & 0.190 & -1.92 & -1.73 & 0.90 \\
\hline NGC 6304 & 1.818 & 0.306 & -0.59 & -0.68 & -1.00 \\
\hline NGC 6342 & 1.771 & 0.375 & -0.62 & -0.69 & -1.00 \\
\hline NGC 6356 & 1.658 & 0.153 & -0.62 & -0.69 & -1.00 \\
\hline NGC 6362 & 1.429 & 0.367 & -1.08 & -0.96 & -0.58 \\
\hline NGC 6388 & 2.130 & 0.121 & -0.74 & -0.74 & -0.67 \\
\hline NGC 6441 & 1.854 & 0.113 & -0.59 & -0.68 & -0.78 \\
\hline NGC 6522 & 1.183 & 0.159 & -1.44 & -1.21 & 0.71 \\
\hline NGC 6544 & 1.500 & 0.395 & -1.56 & -1.32 & 1.00 \\
\hline NGC 6569 & 1.547 & 0.202 & -0.86 & -0.80 & -0.76 \\
\hline NGC 6584 & 1.217 & 0.235 & -1.54 & -1.30 & -0.15 \\
\hline NGC 6624 & 1.605 & 0.243 & -0.35 & -0.63 & -1.00 \\
\hline NGC 6637 & 2.060 & 1.172 & -0.59 & -0.69 & -1.00 \\
\hline NGC 6638 & 1.228 & 0.186 & -1.15 & -0.97 & -0.30 \\
\hline NGC 6642 & 1.319 & 0.265 & -1.29 & -1.08 & 0.29 \\
\hline NGC 6652 & 1.512 & 0.309 & -0.89 & -0.81 & -1.00 \\
\hline NGC 6681 & 1.755 & 0.283 & -1.51 & -1.27 & 0.96 \\
\hline NGC 6717 & 0.722 & 0.267 & -1.32 & -1.10 & 0.98 \\
\hline NGC 6723 & 2.383 & 1.367 & -1.09 & -0.93 & -0.08 \\
\hline NGC 6760 & 1.244 & 0.145 & -0.52 & -0.66 & -1.00 \\
\hline NGC 6864 & 1.712 & 0.194 & -1.32 & -1.10 & -0.07 \\
\hline NGC 6934 & 1.621 & 0.218 & -1.54 & -1.30 & 0.25 \\
\hline NGC 6981 & 1.088 & 0.205 & -1.54 & -1.30 & 0.14 \\
\hline NGC 7078 & 1.883 & 0.175 & -2.15 & -2.12 & 0.67 \\
\hline NGC 7089 & 1.455 & 0.183 & -1.62 & -1.39 & 0.96 \\
\hline NGC 7099 & 2.667 & 0.531 & -2.13 & -1.91 & 0.89 \\
\hline
\end{tabular}




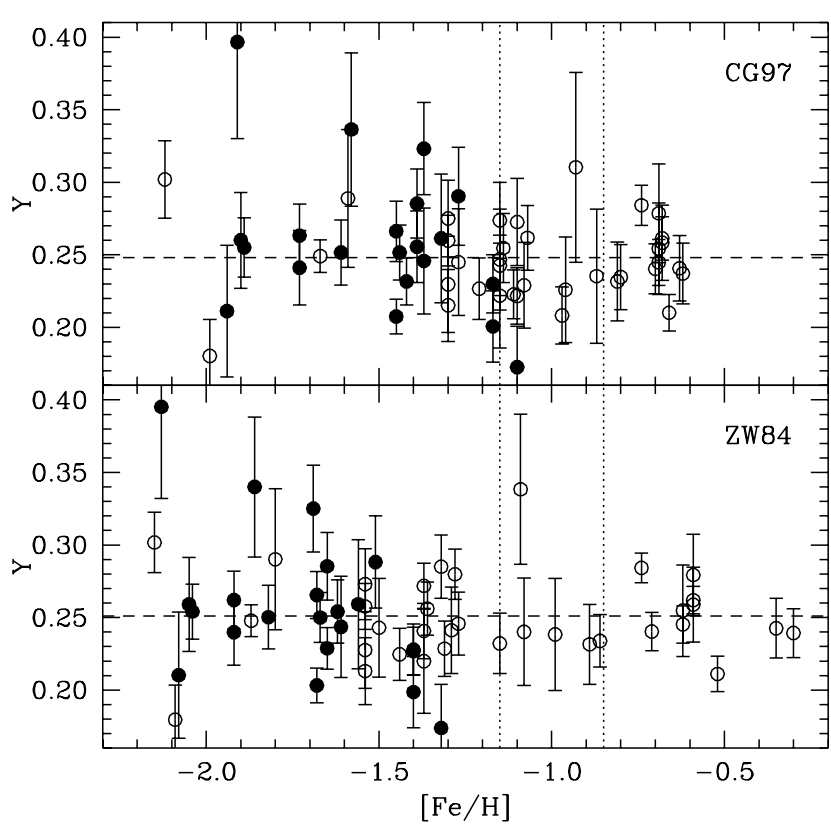

Fig. 2. He abundance values as a function of $[\mathrm{Fe} / \mathrm{H}]$ for the 57 clusters analyzed. The two panels refer to the two metallicity scales adopted in our analysis. The He abundances determined for the clusters with $[\mathrm{Fe} / \mathrm{H}]$ in the range comprised between the two vertical dotted lines are affected by the choice of the cluster age. The horizontal dashed line denotes the most probable value for $Y_{\mathrm{GGC}}$ (see text for details). Filled circles denote clusters with $H B_{\text {type }} \geq 0.8$.

universe (e.g., the WMAP results), we have determined the age of three clusters spanning approximately the entire $[\mathrm{Fe} / \mathrm{H}]$ range of the GGC system, i.e., 47 Tuc, M 3 and M 15; if we consider, e.g., the ZW84 metallicity scale, these clusters span the range between $[\mathrm{Fe} / \mathrm{H}]=-2.10$ and -0.7 . In more detail, we have compared the accurate empirical estimate of the $\Delta V$ parameter values (e.g., the $V$ magnitude difference between the Turn Off and ZAHB level, which is a strong function of the cluster age and weakly affected by metallicity) provided by Rosenberg et al. (1999), with the corresponding values obtained from our isochrones (transformed to the $V$ Johnson photometric system using bolometric corrections to the $V$ band homogeneous with the colour transformations discussed before). We obtained ages of $11.9 \pm 1.2 \mathrm{Gyr}, 11.5 \pm 0.6 \mathrm{Gyr}$, $11.3 \pm 1.1$ Gyr for, respectively, 47 Tuc, M 3 and M 15 . These ages are consistent with a common value between 11 and $12 \mathrm{Gyr}$, and consistent also with an age of the universe of 13.7 Gyr as estimated from the WMAP results. Using the CG97 metallicity scale in place of the ZW84 one has only a small effect on these ages, of less than 1 Gyr. We reiterate again that the exact value of the age assumed for the clusters in our analysis does not influence at all our results about $Y_{\mathrm{GGC}}$.

\subsection{Estimate of $Y_{\mathrm{GGC}}$ using the ZW84 metallicity scale}

We have first considered the ZW84 metallicity scale. The individual $\mathrm{He}$ abundances with associated $1 \sigma$ errors are displayed in the lower panel of Fig. 2. There are 6 clusters in the metallicity range affected by the assumed value of the cluster age; GGCs in this metallicity range do show a large age spread (e.g.,
Rosenberg et al. 1999; Salaris \& Weiss 2002), towards values lower than the common age of the more metal poor objects. This biases the initial He abundance inferred with our assumption of a constant age (13 Gyr) towards lower values, unless the individual cluster age is precisely known and accounted for. These 6 clusters have been therefore excluded from our analysis; when considering the whole remaining sample of 51 clusters, we did not find any statistically significant relationship between $Y$ and $[\mathrm{Fe} / \mathrm{H}]$. We obtain $\delta Y / \delta[\mathrm{Fe} / \mathrm{H}]=-0.01 \pm 0.01$, a ratio different from zero by less than its associated $2 \sigma$ error.

Because there is no dependence of $Y$ on $[\mathrm{Fe} / \mathrm{H}]$, we may assume that any spread in the He abundance among the clusters is not due to Galactic chemical evolution. To investigate the existence of an intrinsic abundance spread uncorrelated with $[\mathrm{Fe} / \mathrm{H}]$ and possible asymmetries (which can skew the best estimate of the initial GGC He abundance when using straight averages or weighted averages) of the $\mathrm{He}$ abundance distribution around the modal value, we have performed the following analysis. Instead of using histograms with the cluster He abundance distribution, we determined a continuous probability distribution function (PDF), following a procedure employed by Sakai et al. (1996) for the luminosity function of RGB stars. More in detail, the He abundance probability density $\Psi(Y)$ for a generic value of $Y$ is determined by replacing the discretely distributed individual abundances by their corresponding Gaussian functions (in the hypothesis of Gaussian errors on the individual abundances), according to the expression

$\Psi(Y)=\sum_{i} \frac{1}{\sigma_{i} \sqrt{2 \pi}} \exp \left(-\frac{\left(Y_{i}-Y\right)^{2}}{2 \sigma_{i}^{2}}\right)$

where $Y_{i}$ and $\sigma_{i}$ are the He abundance and associated error of the $i$ th cluster. $\Psi(Y)$ corresponds to a sum of normalized Gaussian functions, such that a star with smaller error is represented by a Gaussian that is more peaked. If $Y$ is the same for all clusters, the PDF should correspond to a Gaussian function with dispersion $\sigma$ due only to the individual measurement errors.

Figure 3 displays the PDF for the whole sample, with an arbitrary normalization. The peak (i.e. the most probable value of the He abundance for the entire sample) corresponds to $Y=0.251$, and the $1 \sigma$ dispersion of the PDF is equal to 0.038 ; these same values are found when considering the distribution of the individual cluster abundances.

To check if this observed dispersion is compatible with the cluster individual errors we have performed, as in Paper I, the following test. For each individual cluster we have calculated a set of synthetic He abundances by randomly generating - using a Monte Carlo procedure - 10000 abundance values, according to a Gaussian distribution with mean value equal to the observed most probable value, and $\sigma$ equal to the individual He abundance errors. This is repeated for all clusters in the selected sample and the 10000 values for each individual clusters are combined to produce an "expected" distribution for the entire cluster sample, on the assumption that the detected He abundance dispersion is not intrinsic, but due just to the individual errors, assumed Gaussian. The F-test was then applied to determine if this "expected" distribution, which has an 


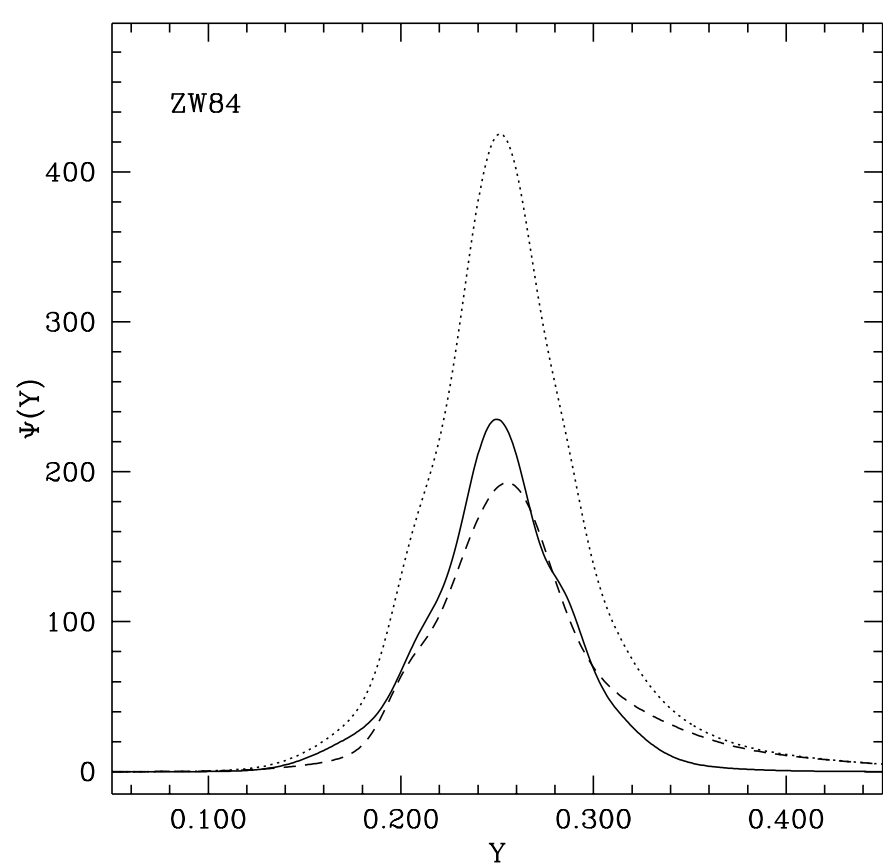

Fig. 3. Probability Distribution Function (PDF) for the He abundance in our GGC sample when using the ZW84 metallicity scale. The dotted line represents the PDF for the 51 clusters with $[\mathrm{Fe} / \mathrm{H}]$ in the range not affected by the cluster age (see text); the solid line represents the subsample with $H B_{\text {type }}<0.8$, while the dashed line represents the subsample with $H B_{\text {type }} \geq 0.8$. The individual PDFs are not normalized, but for each value of $Y$ the sum of the PDFs for the two subsamples selected on the basis of their HB colour is equal to the PDF for the whole sample.

approximately infinite number of elements, displays a variance that is statistically consistent with the observed distribution of 51 objects. We state that a $Y_{\mathrm{GGC}}$ range does exist if the probability that the two distributions have different variance is larger than $95 \%$.

When applying the F-test to our data, we find that this probability is above $99 \%$, therefore we can formally conclude that the observed dispersion is incompatible with the individual error bars. Also the shape of the distribution is different from Gaussian, as shown by the value of the kurtosis, which is equal to 2.8 .

To investigate this matter further, we made a cluster selection on the basis of their HB colour. We considered the value of the ratio $H B_{\text {type }}=(B-V) /(B+V+R)$ (Lee et al. 1994), where $B, V$ and $R$ denote the number of HB stars, respectively, bluer than the RR Lyrae instability strip, inside the strip and redder than the instability strip. The values of $H B_{\text {type }}$ are from the catalogue by Harris (1996), with the exception of NGC 5634, NGC 5946, NGC 6273, NGC 6284, NGC 6388, NGC 6441, NGC 6569, NGC 6642, which are not present in Harris' catalogue. For these clusters we considered the instability strip boundaries of Bono et al. (1995; 1997), and determined the number of stars at the blue and red side of the strip (appropriately reddened according to the individual reddenings given in Harris' catalogue). We then computed the value of $H B_{\text {type }}$ by considering $V$ equal to zero, since we do not have an estimate of the number of RR Lyrae stars for these clusters. In this way we have only an approximate upper limit for the value of $H B_{\text {type }}$, which, as we will see in the following, is enough for our purposes. By considering various sample selections according to the value of $H B_{\text {type }}$, we have determined that for the 30 objects with $H B_{\text {type }}<0.8$ the F-test gives a probability lower than $95 \%$ that there is an intrinsic spread in the initial He abundances. The observed $1 \sigma$ spread - equal to 0.027 - is therefore compatible with the individual errors. Clusters with $H B_{\text {type }}$ in this range (see Fig. 2) still cover the entire $[\mathrm{Fe} / \mathrm{H}]$ interval spanned by the whole sample; again, they do not show any statistically significant trend of $\mathrm{He}$ abundance with $[\mathrm{Fe} / \mathrm{H}]$ $(\delta Y / \delta[\mathrm{Fe} / \mathrm{H}]=0.002 \pm 0.010)$. The He abundance of this sample is therefore formally constant and can be considered to be an estimate of $Y_{\mathrm{GGC}}$. The PDF for this sample - displayed in Fig. 3 - provides a most probable value $Y=0.250$. It is important to notice that the PDF is very symmetric, which is confirmed by the fact that the weighted average of the individual abundances is equal to $0.251 \pm 0.003$, fully consistent with the most probable value provided by the PDF. The kurtosis is equal to only -0.09 , again in agreement with the fact that the PDF can be well approximated by a Gaussian function. If we include in this sample with $H B_{\text {type }}<0.8$ also NGC 5634, NGC 6273 and NGC 6284, for which the computed values of $H B_{\text {type }}=0.91$, 0.97 and 0.83 are only upper limits, the previous results are unchanged.

This result means that the spread found for the whole sample, shown to be inconsistent with the individual measurement errors, must be due to the 21 clusters with $H B_{\text {type }} \geq 0.8$; in fact, they show a $1 \sigma$ abundance spread of 0.05 around the most probable value of the associated PDF $Y=0.255$ (see Fig. 3). This abundance spread is much larger than for clusters with redder $\mathrm{HB}$ colours, in spite of the fact that there is no trend in the error on the individual $\mathrm{He}$ abundances with respect to $H B_{\text {type }}$. The F-test statistics confirms that the abundance spread is inconsistent with the individual measurement errors at the level of more than $99.999 \%$.

If we do not include NGC 5634, NGC 6273 and NGC 6284 in the cluster sample with $H B_{\text {type }} \geq 0.8$, the intrinsic spread is confirmed with the same confidence level.

For the 21 clusters with $H B_{\text {type }} \geq 0.8$ we do not find a correlation between their He abundance and $H B_{\text {type }}$; surprisingly, we obtain a correlation with $[\mathrm{Fe} / \mathrm{H}]$, with a slope significant at more than $2 \sigma$ level, i.e., $\delta Y / \delta[\mathrm{Fe} / \mathrm{H}]=-0.10 \pm 0.04$. This correlation is unexpected because it does not exist for clusters with redder HB types. We will see that it is also absent from the subsample with blue HBs if we use the CG97 [Fe/H] scale, and we believe it is just an artifact due to the uncertainty on the GGC metallicity scale.

It is therefore confirmed that the large abundance spread incompatible with the measurement errors found for the whole sample is due to these clusters with the bluest HB colours. They are the most affected by the possible presence of stellar subpopulations with enhanced $\mathrm{He}$, as well as by the dependence of the HB evolutionary timescale on the stellar mass. Both effects tend to increase the He abundance estimated with our calibration. For these blue HB clusters we find that the peak of the asymmetric PDF is located at an abundance larger than 
the value obtained for clusters with redder $\mathrm{HB}$, which display an abundance spread compatible with the measurement errors. Figure 3 also shows how the high abundance tail of the PDF has more weight than for clusters with redder $\mathrm{HB}$ (the value of the kurtosis is equal to 1.0 for this subsample of blue $\mathrm{HB}$ GGCs). We therefore interpret the $\mathrm{He}$ abundance spread being incompatible with the measurement errors found in this group of blue HB clusters as the effect of the increasing evolutionary timescale for very blue $\mathrm{HB}$ objects, eventually coupled to the presence of He enriched stellar subpopulations. This conclusion is reinforced by the results of the next section.

To avoid these problems with blue HB clusters we will therefore consider - as said before - the sample of 21 clusters with $H B_{\text {type }}<0.8$ for the best estimate of $Y_{\mathrm{GGC}}$. Since the observed spread is compatible with the measurement errors, one possibility - which is what we have implicitly assumed in Paper I - is to consider that the intrinsic dispersion of the individual $Y$ is zero; this assumption is fully compatible with the F-test analysis. In this case the best estimate of $Y_{\mathrm{GGC}}$ is the weighted average (which also agrees with the mode of the associated PDF, as shown before) that provides $Y_{\mathrm{GGC}}=0.251 \pm 0.003$.

Another possibility is that there exists an intrinsic abundance spread not detected by the F-test, because it is masked by the individual errors. We can investigate this issue more deeply by assessing what intrinsic dispersion in the individual He abundances is compatible with the observed distribution of $Y$. Applying the F-test to the actual sample of $Y$ abundances and to synthetic samples computed accounting for the actual individual errors plus increasingly large abundance spreads (in case of Gaussian and uniform intrinsic spread, although of course the mathematical form of the hypothetical abundance spread is unknown), we obtained the somewhat expected result that an intrinsic $1 \sigma$ dispersion up to 0.025 is still allowed by present data. This means that 0.025 is an upper limit to the possible intrinsic spread within our 21 object sample.

As a further test we selected a subsample of 12 clusters with individual errors lower than 0.025 . These clusters span the whole metallicity range covered by the full sample, and their individual He abundances do not show any trend with metallicity. The corresponding abundance distribution shows the same mode and weighted mean as the 21 object sample, and a reduced $1 \sigma$ dispersion of 0.021 , again fully compatible with the individual errors (as assessed through an F-test). Following the same procedure outlined above, we found that the upper limit to the intrinsic $1 \sigma$ spread allowed by this sample is equal to 0.019 . It should be realized that this subsample with smaller individual errors, still containing a sizable number of objects spanning the entire metallicity range, has the same mode and weighted mean and shows an He abundance distribution with a lower dispersion fully compatible with the individual errors. This allows one to safely reduce the upper limit of a possible intrinsic $1 \sigma$ spread to 0.019 .

From our data we can therefore conclude that, if one allows for an intrinsic abundance spread, the most probable value $Y_{\mathrm{GGC}}$ is well represented by the weighted average given above, since it is practically coincident with the mode of the

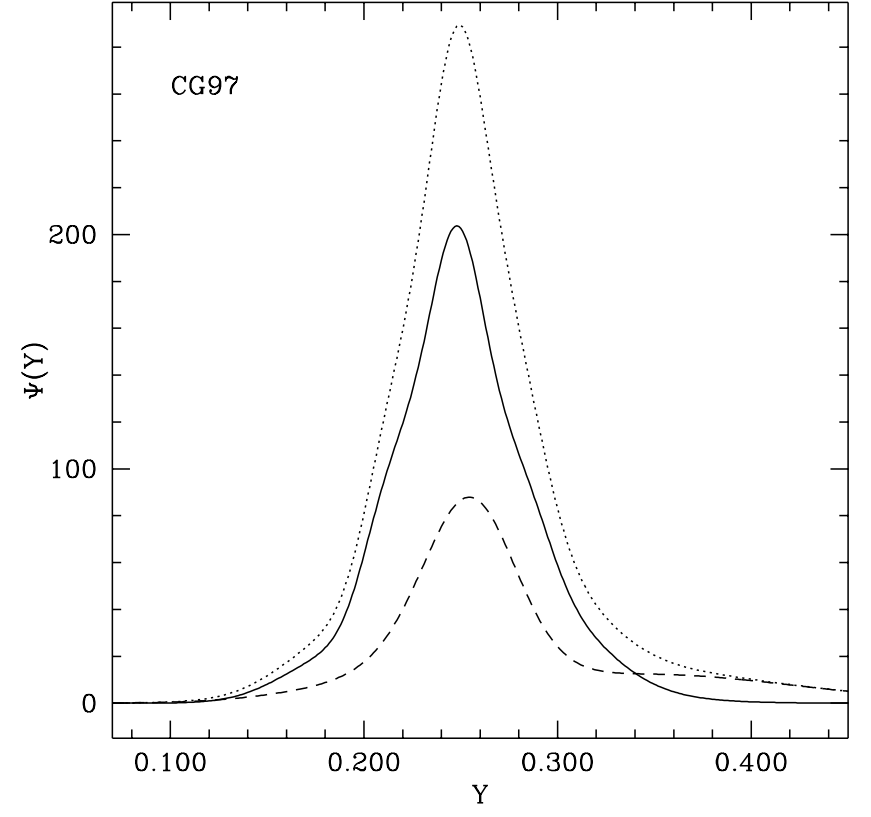

Fig. 4. As in Fig. 3, but for the CG97 [Fe/H] scale (see text).

sample PDF. An upper limit of 0.019 can then be associated to the $1 \sigma$ dispersion around $Y_{\mathrm{GGC}}$ allowed by present data.

\subsection{Estimate of $Y_{\mathrm{GGC}}$ using the CG97 metallicity scale}

We have repeated our abundance analysis considering the CG97 metallicity scale (see Fig. 2). In this case, there are 15 clusters in the metallicity range affected by the cluster age, about $25 \%$ of the whole sample. In the following we will consider only the 42 clusters with $[\mathrm{Fe} / \mathrm{H}]>-0.85$ and $[\mathrm{Fe} / \mathrm{H}]<-1.15$. For these clusters there is again no statistically significant trend of $\mathrm{He}$ abundance with $[\mathrm{Fe} / \mathrm{H}](\delta Y / \delta$ $[\mathrm{Fe} / \mathrm{H}]=-0.017 \pm 0.014)$.

The PDF for the He abundance of this sample is displayed in Fig. 4. The most probable value for the He abundance is $Y=0.250$ and the $1 \sigma$ dispersion is equal to 0.04 . When applying the F-test to this sample, we obtain a statistically significant He abundance spread at more than $99 \%$ confidence level. The shape of the distribution is also very different from a Gaussian one, with a value for the kurtosis equal to 3.1. As for the ZW84 metallicity scale, we find that the 22 clusters with $H B_{\text {type }}<0.8$ do not show a statistically significant He abundance spread, nor a trend of $\mathrm{He}$ abundance with $[\mathrm{Fe} / \mathrm{H}](\delta Y / \delta$ $[\mathrm{Fe} / \mathrm{H}]=-0.002 \pm 0.014)$; for these 22 clusters we obtain a most probable value $Y=0.248$. The PDF is again highly symmetric and the value of the weighted average of the individual abundances, $Y=0.246 \pm 0.004$, fully agrees with the location of the peak of the PDF; the kurtosis is negligible, equal to -0.03 .

For the 20 clusters with $H B_{\text {type }} \geq 0.8$, the abundance spread is statistically significant (at more than $99.999 \%$ confidence level), the most probable value for the He abundance being equal to $Y=0.251$, and there is no trend of the individual abundances with either $[\mathrm{Fe} / \mathrm{H}]$ or $H B_{\text {type }}$. As for the ZW84 metallicities, the bluest $\mathrm{HB}$ clusters have $\mathrm{He}$ abundances shifted to higher values, and again the high $\mathrm{He}$ abundance tail of the PDF 
has substantially more weight than for the case of the clusters with redder HB (the value of the kurtosis is equal to 1.5). The lack of correlation of the individual abundances with $[\mathrm{Fe} / \mathrm{H}]$ for this subsample strongly suggests that the correlation found with ZW84 metallicities is spurious, due to the actual uncertainty on the GGC $[\mathrm{Fe} / \mathrm{H}]$ scale.

As for the ZW84 metallicities, we can consider the subsample of 22 clusters with $H B_{\text {type }}<0.8$ to provide the best estimate of $Y_{\mathrm{GGC}}$. As before, the F-test analysis indicates that either there is no intrinsic spread in the individual He abundances, or some intrinsic spread is masked by the individual measurement errors. In the first case one can use the weighted average $Y_{\mathrm{GGC}}=0.246 \pm 0.004$ as the best estimate of $Y_{\mathrm{GGC}}$. In the second case, following exactly the same steps as for the ZW84 metallicities, we find that the weighted average (again consistent with the mode of the PDF) also provides the most probable value for $Y_{\mathrm{GGC}}$, with a 0.019 upper limit to the $1 \sigma$ dispersion allowed by the present data.

\section{Summary and conclusions}

In this paper we have presented a detailed study of the initial He abundance of the GGC system. We have used the most extensive and homogeneous database of $R$-parameter values to date, and recent updated stellar evolution models. As in Paper I, we do not find any statistically significant correlation between the individual cluster $\mathrm{He}$ abundances and $[\mathrm{Fe} / \mathrm{H}]$. This suggests a very homogeneous value of $Y$ for the GGCs, with practically no He abundance evolution over the entire $[\mathrm{Fe} / \mathrm{H}]$ spanned by the GGC system, at variance with results on the evolution of He in extragalactic HII regions, which measure a gradient of He with Oxygen (hence with $[\mathrm{Fe} / \mathrm{H}]$ ). This result on the evolution of He in GGCs strengthens similar conclusions reached in Paper I, and it has to be taken into account in studies of Galaxy formation mechanisms. Note that Sandquist (2000) also found a constant $Y$ for the GGC system using two independent indicators of relative $\mathrm{He}$ abundances, namely the $\Delta$ parameter (difference in magnitude between HB and Main Sequence) and the RR Lyrae mass-luminosity exponent $A$.

As for the existence of an intrinsic spread of the cluster initial $Y$, we found that objects with $H B_{\text {type }}<0.8$ - irrespective of their $[\mathrm{Fe} / \mathrm{H}]$ - do show a remarkably homogeneous and symmetric abundance distribution, whose spread is fully compatible with the measurement errors. One can therefore conclude that either the He abundance is the same for all clusters, without any intrinsic dispersion, or that an eventual dispersion is masked by the individual errors. In this case we obtain that our data allow a 0.019 upper limit to a possible $1 \sigma$ intrinsic dispersion. On the other hand, clusters with blue HB colours $\left(H B_{\text {type }} \geq 0.8\right)$ show a spread in their initial $Y$ incompatible with the individual error bars, skewed towards values higher than those for clusters with redder HB. We interpret this result as due to the increase of evolutionary timescales along the HB phase for progressively bluer HB stars, which causes an overestimate of $Y$ when using our calibration. This effect may be coupled to the possible presence of a subpopulation of He enhanced stars in at least some GGCs (see D’Antona et al. 2002), which also leads to an overestimate of $Y$ (at most by $0.01-0.02$ ) when determined from our calibration.

As already noticed, for clusters with $H B_{\text {type }}<0.8$ we obtain a distribution of $Y$ values very close to Gaussian and a $1 \sigma$ dispersion consistent with the measurement errors. This occurrence goes against the possible existence of a sizable spread towards higher $Y$ values due to subpopulations of He enriched stars, as in blue HB clusters; in fact, in case redder $\mathrm{HB}$ clusters, the effect of these subpopulations is at most at the level of $\sim 0.005$, and probably smaller. It is also reasonable to assume that these subpopulations might not be present in all clusters, and the net effect on our estimates of $Y_{\mathrm{GGC}}$ should therefore be negligible. An extreme possibility is that all clusters with $H B_{\text {type }}<0.8$ are affected in the same way by this enhancement, whose maximum effect would be to bias our $Y_{\mathrm{GGC}}$ estimate by $\sim 0.005$ towards too high values.

We conclude by providing a best estimate for $Y_{\mathrm{GGC}}$, including the effect of the still uncertain $[\mathrm{Fe} / \mathrm{H}]$ scale and the sources of systematic errors discussed in Paper I (i.e., the error on the ${ }^{12} \mathrm{C}(\alpha, \gamma){ }^{16} \mathrm{O}$ reaction rate and the method adopted to suppress the breathing pulses during the final stages of central He burning). We have considered as reference value the $Y_{\mathrm{GGC}}$ abundance determined with the ZW84 $[\mathrm{Fe} / \mathrm{H}]$ scale. Starting with this reference $Y_{\mathrm{GGC}}$ we have generated a set of 100000 synthetic He-abundance values, by applying (through a Monte Carlo simulation) to the reference value a set of random and systematic errors, according to a given probability distribution. In particular, random errors have been modeled according to a Gaussian distribution with mean value equal to the reference one, and $1 \sigma$ dispersion equal to the corresponding random error on $Y_{\mathrm{GGC}}$. The systematic uncertainties due to the choice of the $[\mathrm{Fe} / \mathrm{H}]$ scale (which causes a decrease of $Y_{\mathrm{GGC}}$ by 0.005 with respect to the reference value), ${ }^{12} \mathrm{C}(\alpha, \gamma){ }^{16} \mathrm{O}$ reaction rate (variation by \pm 0.008 ), and breathing pulses suppression technique (increase by 0.003 ) have been modeled using a uniform distribution spanning the appropriate range.

The mean value for the final synthetic distribution of He abundances is $Y_{\mathrm{GGC}}=0.250 \pm 0.006$. An intrinsic dispersion with a firm $1 \sigma$ upper limit of 0.019 around this value of $Y_{\mathrm{GGC}}$ is a priori possible given the observational errors. This estimate of $Y_{\mathrm{GGC}}$ is in good agreement with the primordial He abundance inferred from the CMB in conjunction with Big-Bang nucleosynthesis computations. Within the respective $1 \sigma$ errors this value is also in agreement with the results from Paper I.

Acknowledgements. We warmly thank R. Gratton, G. Steigman and A. Irwin for interesting discussions on this topic and useful suggestions, and an anonymous referee for very pertinent remarks. M.S. wishes to thank past and present Rockets for the inspiration they provided during all these years, and Toby Moore for useful discussions. This work has been partially supported by the Italian Ministero dell'Istruzione e della ricerca (PRIN2002 and PRIN2003) and by the Agenzia Spaziale Italiana.

\section{References}

Alexander, D. R., \& Ferguson, J. W. 1994, ApJ, 437, 879

Angulo, C., Arnould, M., Rayet, M., et al. 1999, Nucl. Phys. A, 656, 3

Bessel, M. S., Castelli, F., \& Plez, B. 1998, A\&A, 333, 231 
Bono, G., Caputo, F., \& Marconi, M. 1995, AJ, 110, 2365

Bono, G., Caputo, F., Cassisi, S., Incerpi, R., \& Marconi, M. 1997, ApJ, 483, 811

Burles, S., Nollett, K. M., \& Turner, M. S. 2001, ApJ, 552, L1

Buzzoni, A., Fusi Pecci, F., Buonanno, R., \& Corsi, C. E. 1983, A\&A, 128,94

Caputo, F., Martinez Roger, C., \& Paez, E. 1987, A\&A, 183, 228

Cassisi, S., Castellani, V., degl'Innocenti, S., \& Weiss, A. 1998, A\&AS, 129, 267

Cassisi, S., Salaris, M., \& Irwin, A. W. 2003, ApJ, 588, 862

D’Antona, F., Caloi, V., Montalban, J., Ventura, P., \& Gratton, R. G. 2002, A\&A, 395, 69

Harris, W. E. 1996, AJ, 112, 1487

Iben, I. Jr. 1968, Nature, 220, 143

Iglesias, C. A., \& Rogers, F. J. 1996, ApJ, 464, 943

Izotov, Y. I., \& Thuan, T. X. 1998, ApJ, 497, 227

Izotov, Y. I., \& Thuan, T. X. 2003, ApJ, in press [arXiv: astro-ph/0310421]

Kunz, R., Fey, M., Jaeger, M., et al. 2002, ApJ, 567, 643

Lee, Y.-W., Demarque, P., \& Zinn, R. 1994, ApJ, 423, 248
Michaud, G., Vauclair, G., \& Vauclair, S. 1983, ApJ, 267, 256

Ödman, C. J., Melchiorri, A., Hobson, M. P., \& Lasenby A. N. 2002, Phis. Rev. D, 67, 083511

Origlia, L., \& Leitherer, C. 2000, AJ, 119, 2018

Olive, K. A., Skillman, E. D., \& Steigman, G. 1997, ApJ, 483, 788

Piotto, G., King, I. R., Djorgovski, S. G., et al. 2002, A\&A, 391, 945 Potekhin, A. Y. 1999, A\&A, 351, 787

Pryke, C., Halverson, N. W., Leitch, E. M., et al. 2002, ApJ, 568, 46

Riello, M., Cassisi, S., Piotto, G., et al. 2003, A\&A, 410, 553

Rosenberg, A., Saviane, I., Piotto, G., \& Aparicio, A. 1999, AJ, 118, 2306

Sakai, S., Madore, B. F., \& Freedman, W. L. 1996, ApJ, 461, 713

Salaris, M., Cassisi, S., \& Weiss, A. 2002, PASP, 114, 375

Salaris, M., \& Weiss, A. 1998, A\&A, 335, 943

Salaris, M., \& Weiss, A. 2002, A\&A, 388, 492

Sandage, A., \& Wildey, R. 1967, ApJ, 150, 469

Sandquist, E. L. 2000, MNRAS, 313, 571

Sievers, J. L., Bond, J. R., Cartwright, J. K., et al. 2003, ApJ, 591, 599

Spergel, D. N., Verde, L., Peiris, H. V., et al. 2003, ApJS, 148, 175

Zoccali, M., Cassisi, S., Bono, G., et al. 2000, ApJ, 538, 289 